\title{
Visual evoked cortical potentials and pattern electroretinograms in Parkinson's disease and control subjects
}

\author{
S NIGHTINGALE, ${ }^{*} \mathrm{~K}$ W MITCHELL, $\dagger \mathrm{J}$ W HOWE $\ddagger$ \\ From the Departments of Neurology* and Medical Physics, $\uparrow$ Royal Victoria Infirmary, and the University \\ Department of Ophthalmology, $\ddagger$ University of Newcastle upon Tyne, Newcastle upon Tyne, UK
}

SUMMARY Parkinson's disease patients have been shown to have abnormal visually evoked cortical potentials (VEPs) to pattern stimulation. Whereas dopamine is not an important neurotransmitter in the central visual pathways, the retina is rich in dopamine and, together with previous animal and human studies, this suggests that the abnormal VEPs in Parkinson's disease patients may be due to a biochemical and electrophysiological disorder in the retina. This hypothesis has been examined by studying the VEPs and pattern electroretinogram (PERG) of Parkinson's disease patients and matched control subjects. The amplitudes of the cortical and retinal evoked potentials were significantly reduced in Parkinson's disease patients compared with the control subjects and this could not be attributed to any particular feature of the disease or its treatment. There was a significant relationship between the VEP P100 latency and the PERG amplitude. Moreover for those subjects in whom there was an interocular difference in both cortical and retinal evoked potentials, the abnormality was more commonly found in the potentials from the same eye. These findings suggest that the abnormality of the VEP in Parkinson's disease patients is, at least in part, secondary to an abnormality of the retina itself.

The measurement of the visually evoked cortical potential (VEP) is a widely used non-invasive technique for studying the visual system and its most important clinical application is in the assessment of multiple sclerosis. However, changes in the VEP are found in a variety of other central nervous system disorders, ${ }^{1}$ including Parkinson's disease. ${ }^{2-6}$ It is perhaps surprising that there is a delay in the VEP in Parkinson's disease, a disorder of the motor system in which there is no obvious clinical involvement of the visual system and with no pathological lesions, such as demyelination, that might account for the delays in conduction. Although the increased latency of the VEP in multiple sclerosis has in general been attributed to demyelination in the visual pathways, other mechanisms such as humoral factors, synaptic malfunction or changes in dendritic potentials may play a

Address for reprint requests: Dr S Nightingale, Midland Centre for Neurosurgery and Neurology, Holly Lane, Smethwick, Birmingham, B67 7JX, UK.

Received 15 August 1985 and in revised form 7 January 1986. Accepted 13 January 1986 part. Such mechanisms may also be relevant to central nervous system disorders other than multiple sclerosis which have abnormal VEPs. Although the major clinical manifestations of Parkinson's disease involve the motor systems and the responsible pathology is located in the basal ganglia, there is evidence of more widespread disease, both pathologically, ${ }^{7}$ electrophysiologically ${ }^{4}$ and clinically. ${ }^{5}$ In particular, Kupersmith et $\mathrm{l}^{5}$ have demonstrated reduced visual contrast sensitivity in Parkinson's disease patients compared with matched controls. There is ample evidence in animals and man that changes in neurotransmitters alone can alter the VEP latency and amplitude ${ }^{8-10}$ and it seems likely that the abnormal VEP in Parkinson's disease is produced in this way. However, the site in the visual system at which neurotransmitter changes might influence the VEP is unknown as is the mechanism by which this influence might be exerted.

The fact that dopamine is found in the interplexiform layer of the retina of vertebrates including man, ${ }^{11}$ but is not known to be an important neurotransmitter elsewhere in the visual system, suggests a retinal origin for the abnormality in the VEP. More- 
over, in cat and rat retina, changes in dopamine metabolism occur with alterations in illumination. ${ }^{12-14}$ The interocular difference in the VEP latency in some Parkinson's disease patients ${ }^{4}$ also suggests a prechiasmal, possibly retinal, lesion. Further evidence of a retinal origin for the abnormal VEP produced by altered transmitter levels is provided in studies by Dyer et $a l^{8}$ on the delayed flash VEP in hooded rats after dopamine depletion had been achieved neuropharmacologically. In these rats the VEP following direct optic nerve stimulation was of normal latency whereas the flash-evoked potential recorded directly from the optic nerve was delayed, indicating that the abnormality responsible for the delay in VEP to a flash stimulus was situated anterior to the optic nerve, most probably in the retina.

Attempts to assess retinal physiology in Parkinson's disease have also been made. The function of the retinal pigment epithelium has been studied by means of the electro-oculogram technique (EOG) in Parkinson's disease patients, who had reduced EOG ratios and following levodopa therapy the ratio returned to control values. ${ }^{15}$ The function of more proximally situated components of the retina have been studied by Cavallacci and Perossini ${ }^{16}$ in Parkinson's disease patients using the conventional flash electroretinogram (flash ERG), but no abnormalities were found apart from a reduction in the amplitude of the " $b$ " wave in some Parkinson's disease patients who were overdosed with levodopa. Kupersmith $e t a l^{5}$ specifically studied the oscillatory potentials of the flash ERG in Parkinson's disease patients, since these potentials are thought to be generated by the inner nuclear layer, ${ }^{1718}$ where the dopaminecontaining cells reside. However, no difference in the oscillatory potentials between Parkinson's disease patients and controls were found. Since the flash ERG is a mass retinal response to which dopaminecontaining cells would only contribute a small part, subtle changes in retinal physiology may be missed by this technique. Moreover the VEP is largely a measure of macular function, whereas the whole retina contributes to the relatively crude flash ERG response. For these reasons the flash ERG, though the conventional method of studying retinal physiology, is not suitable for assessing possible retinal abnormalities in Parkinson's disease patients.

We have chosen to study the pattern electroretinogram ${ }^{19}$ (PERG) in association with the VEP in Parkinson's disease patients. Whereas the flash ERG reflects the function of distal retinal layers from a wide retinal area, current research suggests that the PERG is generated in more proximal retinal layers which include the retinal ganglion cells. ${ }^{20-22}$ The flash ERG is a useful method of studying abnormalities of photoreceptor function and dark adapta- tion, but little other information is obtained about retinal physiology; indeed the flash ERG may, on occasions, bear little relationship to visual function. For example, it may be normal in the presence of severe visual impairment from retinal lesions such as macular dystrophy, and conversely may be abnormal in patients with photoreceptor/RPE disorders while central visual function remains relatively intact. In contrast, the PERG is often abnormal when vision is impaired due to disorders of the central retina and optic nerve. ${ }^{23-29}$ The PERG may therefore be particularly relevant to the investigation concerning a retinal origin of an abnormal VEP in Parkinson's disease patients. Furthermore, the PERG and VEP can be simultaneously recorded under identical stimulus conditions ${ }^{272830-33}$ and this approach has been adopted in the present study.

\section{Patients and methods}

Thirty-six patients with idiopathic Parkinson's disease were studied (male 19; female 17). All were attending out-patients at the Department of Neurology at the Royal Victoria Infirmary in Newcastle upon Tyne. The severity of the Parkinson's disease was graded by an overall motor function into Mild (Grade 1) when the clinical picture provided little or no functional disability; Moderate (Grade 2) when there was some troublesome interference with motor function though most activities could still be performed; Severe (Grade 3) when a variety of everyday activities could no longer be performed; Very Severe (Grade 4) when the patient was dependent on others for most or all of his needs. Four patients were in grade 1, sixteen in Grade 2, thirteen in Grade 3 and three in Grade 4 . Twenty-seven patients were taking drugs containing levodopa; five were also on anticholinergic drugs and two also on bromocriptine. Four patients exhibited the "on/off" phenomenon; fourteen patients had chorea. The 28 control subjects (male 14; female 14) were drawn from in-patients at the Royal Victoria Infirmary, mainly from the Departments of Orthopaedic Surgery and Dermatology. Informed consent was obtained in all cases.

All subjects (Parkinson's disease and control) were free of ophthalmic or other neurological disorders and all had corrected visual acuity better than 6/36 in each eye. The Parkinson's disease patients and control subjects were well matched for age (Parkinson's disease mean age 62.6 years, SD 5.3; control mean age $62 \cdot 3$ years, SD 6.9).

In each patient the corrected visual acuity was recorded, and an ophthalmoscopic examination made in order to exclude any ocular pathology which may have influenced the results. Visual acuity was expressed in a decimal notation in which $6 / 60$ is $0 \cdot 1$ and $6 / 6$ is 1.0 .

The Flash and Pattern ERGs were obtained using Arden gold foil electrodes with a silver/silver chloride disc reference electrode placed over the outer canthus. An earth electrode was attached to the mid-frontal area. The VEP was recorded from scalp electrodes placed on the following positions: ${ }^{34}$ Active: $\mathrm{Oz}$, Reference: $\mathrm{Cz}$. A Ganzfeld stimulator was used to obtain the photopic flash ERG. The control subjects were 

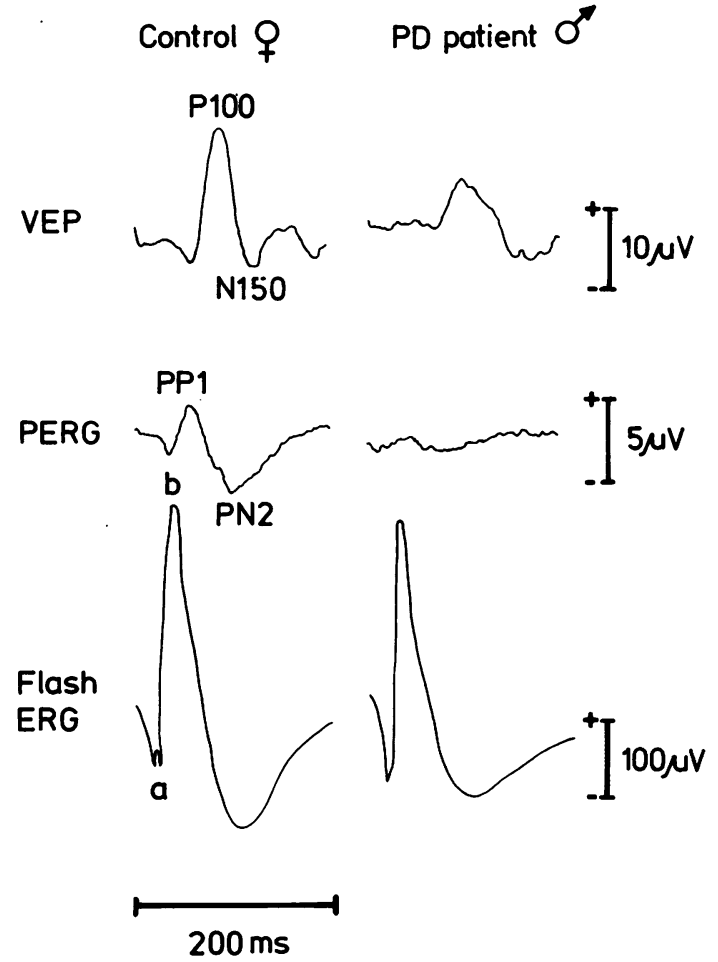

Fig 1 Examples of flash ERG, PERG and VEP obtained from a control female subject and a Parkinson's disease male patient. Stimulus checksize 50'. Flash ERG stimulus 1.0 joules.

studied at intensities of both $0 \cdot 1$ joule and $1 \cdot 0$ joule. Twentyfive Parkinson's disease patients were studied using the higher intensity and eleven using the lower.

The PERG and VEPs were generated using a high quality TV monitor producing a pattern-reversing checkerboard under the following conditions:

Contrast $-95 \%$

Check Subtense-25' \& 50'

Reversal rate- $2 \mathrm{~s}^{-1}$

Mean luminance- $12 \mathrm{Cd} . \mathrm{m}^{-2}$

Field Size $-17^{\circ} \times 14^{\circ}$

One hundred and twenty-eight stimulus epochs of $200 \mathrm{~ms}$ were obtained in each average and the amplifier bandwidth was selected to be $0 \cdot 8-80 \mathrm{~Hz}$. A LED (light emitting diode) was attached to the centre of the screen to facilitate fixation which was monitored independently using closed circuit TV. It was a pre-requisite that adequate fixation could be maintained throughout the investigation for inclusion in the series. Potentials contaminated by eye movement or blink artifact were automatically rejected from the final average signal. Patients who had "on/off" phenomenon were studied during the "off" phase, which is to say when they exhibited Parkinsonian signs rather than chorea. In ten Parkinson's disease patients, the evoked potentials were repeated using the same method and very similar recordings were obtained.

The peak-to-peak amplitude of the " $a$ "-" $b$ " components in the flash ERG was measured. A typical PERG (fig 1) consists of an early negative component (Pattern Negative 1-PN1) of implicit time $30 \mathrm{~ms}$, succeeded by a positive component (Pattern Positive 1-PP1) at $60 \mathrm{~ms}$, and a later after wave (PN2) at about $100-110 \mathrm{~ms}$. In this study the peak-topeak amplitudes of the PP1-PN2 components were measured as were their implicit times. The VEP was analysed with respect to the peak-peak amplitude of the P100-N150 components and their corresponding latencies (fig 1). The tracings were randomised and the measurements made in a blind manner by one observer (KWM).

The groups were compared by Student's $t$ test; a pooled variance estimate was employed unless the standard deviations of the two groups were significantly different $(p<$ 0.05 ), in which case a separate variance estimate was used. Visual acuity and PERG amplitude were not normally distributed and for these variables a Mann-Whitney $U$ test was employed. Correlation between variables was assessed by Pearson's product-moment correlation coefficient, except for PERG amplitude when Spearman's correlation coefficient (rho) was used.

\section{Results}

The following results of the visual acuity and evoked potential measurements are based on the average values of the left and right eyes of each subject with the exception of the results presented in the section on interocular differences. When low amplitude or gross deformation of the recorded evoked potential prevented measurement of latency or implicit time, the case is excluded from the relevant analyses. The slightly differing numbers of cases in the following results reflect these missing values. In addition three Parkinson's disease patients (two female; one male) were unable to tolerate the Ganzfeld stimulator and no flash ERG was recorded.

\section{Visual acuity}

No significant difference was found between the visual acuity of the Parkinson's disease patients and the control subjects (Parkinson's disease mean 0.60, SD 0.30; Control mean 0.72, SD 0.30), nor between the males and females (Males mean 0.62, SD 0.33; Females mean 0.66, SD 0.28).

\section{VEP amplitude}

There was a significant difference in amplitude of the VEP between the Parkinson's disease patients and controls, both at $50^{\prime}$ and $25^{\prime}$ checksize (table 1). However, this difference was largely due to differences in amplitude between female Parkinson's disease patients and female control subjects, whereas the amplitude of the male controls differed little from that of the male Parkinson's disease patients. There was a highly significant difference between the males and 
Table 1 VEP amplitudes

\begin{tabular}{|c|c|c|c|c|c|c|}
\hline$V E P$ & Checksize & $M / F$ & Controls & & Parkinson's disease & All \\
\hline \multirow[t]{2}{*}{ Ampl. } & $50^{\prime}$ & $\begin{array}{l}\mathbf{F} \\
\mathbf{M} \\
\text { All }\end{array}$ & $\begin{array}{l}17 \cdot 4(4 \cdot 3 ; 14) \\
p=0 \cdot 003 \\
11 \cdot 2(5 \cdot 5 ; 14) \\
14 \cdot 3(5 \cdot 8 ; 28)\end{array}$ & $\begin{array}{l}p=0.004 \\
(p=0.06) \\
p=0.002\end{array}$ & $\begin{array}{c}12 \cdot 6(4 \cdot 0 ; 17) \\
\mathrm{p}<0.001 \\
8 \cdot 0(2 \cdot 5 ; 19) \\
10 \cdot 2(4 \cdot 0 ; 36)\end{array}$ & $\begin{array}{l}14 \cdot 8(4 \cdot 7 ; 31) \\
\mathrm{p}<0.001 \\
9 \cdot 4(4 \cdot 3 ; 33) \\
-\end{array}$ \\
\hline & $25^{\prime}$ & $\begin{array}{l}\mathbf{F} \\
\mathbf{M} \\
\text { All }\end{array}$ & $\begin{array}{l}16.9(3.5 ; 13) \\
p=0.002 \\
10.6(5.4 ; 14) \\
13.6(5.5 ; 27)\end{array}$ & $\begin{array}{l}p<0.001 \\
p=0.036 \\
p<0.001\end{array}$ & $\begin{array}{l}10.9(3.7 ; 17) \\
\mathrm{p}=0.001 \\
7.0(2.7 ; 18) \\
8.9(3.7 ; 35)\end{array}$ & $\begin{array}{c}13 \cdot 5(4 \cdot 7 ; 30) \\
\mathrm{p}<0.001 \\
8 \cdot 6(4 \cdot 4 ; 32) \\
-\end{array}$ \\
\hline
\end{tabular}

Results for each group expressed in the form MEAN (STANDARD DEVIATION; NUMBER OF SUBJECTS). Details of the statistical methods are described in the methods section.

females among both the Parkinson's disease patients and control subjects.

\section{VEP latency}

The latency of the P100 and N150 components were not significantly different between the Parkinson's disease patients and control subjects at either checksize, nor did separating males from females demonstrate any significant difference between Parkinson's disease patients and controls. The P100 latency of the male subjects (mean $=122.3 \mathrm{~ms}, \mathrm{SD}=9.9, \mathrm{~N}=33$ ) was longer than that of the female subjects (mean = $116.5 \mathrm{~ms}, \mathrm{SD}=9.3, \mathrm{~N}=31 ; \mathrm{p}=0.019$ ) at $50^{\prime}$ checksize and similar results were obtained with $25^{\prime}$ checksize (male mean $=125 \cdot 2 \mathrm{~ms}, \mathrm{SD}=10 \cdot 6, \mathrm{~N}=32$; female mean $=117.7 \mathrm{~ms}, \mathrm{SD}=7.2, \mathrm{~N}=30 ; \mathrm{p}=$ 0.002 ). The latency of the $N 150$ component was also significantly longer for the males than the females at both $50^{\prime}(\mathrm{p}=0.008)$ and $25^{\prime}$ checksize $(\mathrm{p}=0.011)$. The temporal dispersion of the VEPs (calculated as the latency difference between the P100 and the N150) was no different in the Parkinson's disease patients and control subjects at either checksize. There was a significant inverse correlation between the VEP P100 latency and the VEP amplitude for all subjects at $50^{\prime}$ $(\mathrm{r}=-0.28, \mathrm{p}=0.01)$ and at $25^{\prime}$ checksize $(\mathrm{r}=$ $-0.27, p=0.01$ ). For Parkinson's disease patients alone, the correlation coefficient was -0.37 ( $\mathrm{p}=$ $0.014)$ at $50^{\prime}$ and $-0.29(\mathrm{p}=0.04)$ at $25^{\prime}$ checksize, but for control subjects no significant correlation was observed. For all subjects or for Parkinson's disease patients alone, partial correlation of VEP P100 latency and VEP amplitude controlling for age did not affect these significant relationships, but controlling for sex did abolish the inverse correlation. The inverse correlation of the VEP P100 latency and VEP amplitude can therefore be partly explained by the fact that female subjects tend to have shorter latencies and greater amplitudes whereas in males the opposite is observed.

\section{PERG amplitude}

The PERG amplitude was significantly higher in con- trol subjects than Parkinson's disease patients at 50' checksize (control mean $=5.01 \mu \mathrm{V}, \mathrm{SD}=2.2, \mathrm{~N}=$ 28: Parkinson's disease mean $=3 \cdot 77 \mu \mathrm{V}, \mathrm{SD}=2 \cdot 3$, $\mathrm{N}=36 ; \mathrm{p}=0.03)$. For males alone the difference was significant (control mean $=5 \cdot 36 \mu \mathrm{V}, \mathrm{SD}=2 \cdot 3$, $\mathrm{N}=14$; Parkinson's disease mean $=3.63 \mu \mathrm{V}, \mathrm{SD}=$ $2 \cdot 4, \mathrm{~N}=19 ; \mathrm{p}=0.047$ ), but not for females alone. Although the means of the PERG amplitude to $25^{\prime}$ checksize were greater in the control subjects than in the Parkinson's disease patients for both sexes, there was considerable overlap of the distributions and the differences were not statistically significant.

\section{PERG implicit time}

There was no consistent difference in the implicit time of the PP1 or PN2 wave at either checksize between Parkinson's disease patients and controls. No consistent significant differences were observed between the sexes. There was a significant inverse correlation for all subjects between the PERG amplitude and PP1 implicit time at $50^{\prime}(\mathrm{r}=-0.36 ; \mathrm{p}=0.003)$ and $25^{\prime}$

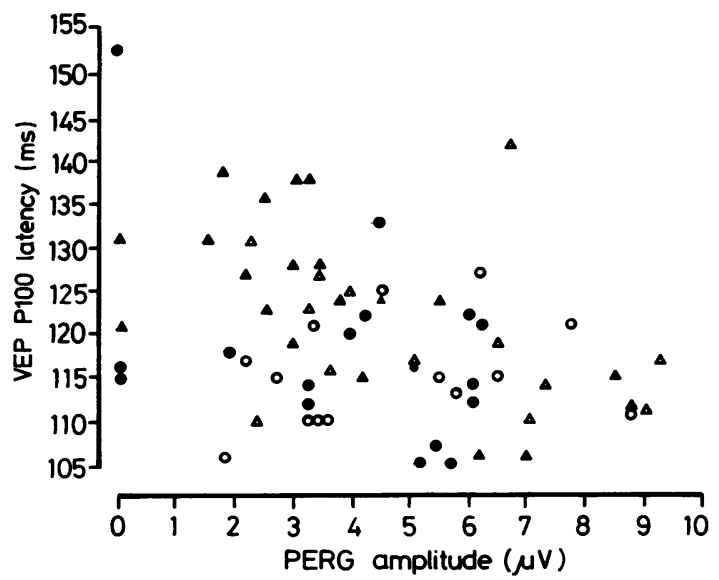

Fig 2 Scattergram of VEP P100 latency with PERG amplitude. Stimulus checksize $50^{\prime} . \bigcirc=$ female; $\triangle=$ male; open symbol = control subject; closed symbol = Parkinson's disease patient. 
checksize $(r=-0.25 ; p=0.04)$ and neither correlation was affected by controlling for the effect of age, sex or the presence of Parkinson's disease. The correlation was also significant for the Parkinson's disease patients alone at $50^{\prime}(\mathrm{r}=-0.59 ; \mathrm{p}<0.001)$ and $25^{\prime}$ checksize $(\mathrm{r}=-0.33 ; \mathrm{p}=0.04)$ and males alone at $50^{\prime}(\mathrm{r}=-0.59 ; \mathrm{p}=0.001)$ and $25^{\prime}$ checksize $(r=-0.48 ; p=0.004)$, but not for the females or controls alone.

\section{Relationship between cortical and retinal potentials}

The relationships between the VEP P100 latency and the PERG amplitude of all subjects at $50^{\prime}$ checksize are shown in fig 2 . There is a highly significant inverse correlation $(\mathrm{r}=-0.38 ; \mathrm{p}=0.001)$. The association is also significant for each of the groups comprising Parkinson's disease patients $(r=-0.53 ; p<0.001)$ or male subjects $(\mathrm{r}=-0.55 ; \mathrm{p}<0.001)$. In particular, male Parkinson's disease patients who are the group with the most abnormal cortical and retinal potentials showed a close correlation $(r=-0.67 ; p$ $=0.001$ ). The relationship of the VEP P100 latency and the PERG amplitude at $25^{\prime}$ checksize showed similar correlations in these subjects groups (all subjects $r=-0.4 ; p=0.001$; Parkinson's disease patients $r=-0.53 ; p=0.001$; males $r=-0.57 ; p$ $<0.001)$. There was little scatter among the control subjects or among female patients generally and for these groups the correlation was not significant. For all subjects or for Parkinson's disease patients alone, partial correlation of VEP P100 latency with PERG amplitude controlling for the effect of age, sex or presence of Parkinson's disease did not affect these significant inverse correlations; indeed controlling for the effect of sex increased the correlation coefficient.

Flash ERG

A significant difference $(\mathrm{p}<0.001)$ in the amplitude

Table 2 Correlation of age with VEP, PERG and Flash ERG. (Parkinson's disease patients and controls combined)

\begin{tabular}{|c|c|c|c|c|c|}
\hline & & Check & $N$ & $r$ & $p$ \\
\hline VEP & $\begin{array}{l}\text { P100 } \\
\text { N150 } \\
\text { Ampl. }\end{array}$ & $\begin{array}{l}50^{\prime} \\
25^{\prime} \\
50^{\prime} \\
25^{\prime} \\
50^{\prime} \\
25^{\prime}\end{array}$ & $\begin{array}{l}64 \\
64 \\
62 \\
63\end{array}$ & $\begin{array}{l}0.41 \\
0 \cdot 48 \\
0 \cdot 35 \\
0 \cdot 43\end{array}$ & $\begin{array}{l}p<0.001 \\
p<0.001 \\
p=0.002 \\
p<0.001 \\
\text { NS } \\
\text { NS }\end{array}$ \\
\hline PERG & $\begin{array}{l}\text { PP1 } \\
\text { PN2 } \\
\text { Ampl. }\end{array}$ & $\begin{array}{l}50^{\prime} \\
25^{\prime} \\
50^{\prime} \\
25^{\prime} \\
50^{\prime}\end{array}$ & $\begin{array}{l}57 \\
51 \\
56 \\
64\end{array}$ & $\begin{array}{r}0 \cdot 31 \\
0 \cdot 40 \\
0 \cdot 23 \\
-0 \cdot 34\end{array}$ & $\begin{array}{l}\mathrm{p}=0.01 \\
\mathrm{p}=0.002 \\
\mathrm{p}=0.04 \\
\mathrm{NS} \\
\mathrm{p}=0.003\end{array}$ \\
\hline Flash E & & $\begin{array}{l}25^{\prime} \\
\text { Ampl. }\end{array}$ & 63 & -0.44 & $\begin{array}{l}\mathrm{p}<0.001 \\
\mathrm{NS}\end{array}$ \\
\hline
\end{tabular}

$\mathrm{N}=$ number of subjects. $\mathrm{r}=$ correlation coefficient. The statistical methods are described in the methods section. NS = not significant. of the flash ERG to 1.0 joule flash stimulation between the Parkinson's disease patients (mean = $96 \mu \mathrm{V}, \mathrm{SD}=52, \mathrm{~N}=22$ ) and control subjects (mean $=146 \mu \mathrm{V}, \mathrm{SD}=40, \mathrm{~N}=28$ ) was observed. No significant differences were noted between the sexes. The amplitude of the flash ERG to $0 \cdot 1$ joule and the implicit time at either stimulus intensity showed no significant differences between Parkinson's disease patients and control subjects or between the sexes. Although for all subjects combined there was a correlation between the flash ERG amplitude to 1.0 joule and the VEP amplitude to $50^{\prime}(\mathrm{r}=0.42 ; \mathrm{p}=0.001)$ and to $25^{\prime}$ checksize $(r=0.44 ; p=0.001)$, no correlation was observed in the Parkinson's disease patients alone. Controlling for the effect of age or sex had little effect on this significant association, but controlling for the presence of Parkinson's disease reduced but did not abolish it. No correlation was demonstrated between flash ERG and PERG for any amplitude or implicit time measure in any subject group.

\section{Effect of severity, treatment and side effects of} treatment in Parkinson's disease patients

When the Parkinson's disease patients were divided by severity into mild (grade 1 and 2) or severe (grade 3 and 4) no significant differences were observed between the groups in any of the amplitude, latency or implicit time measurements of either cortical or retinal potentials. When either group was compared with the control subjects there were no significant differences other than those obtained by comparing all the Parkinson's disease patients to control subjects (described above). Similarly no significant differences were found when the Parkinson's disease patients

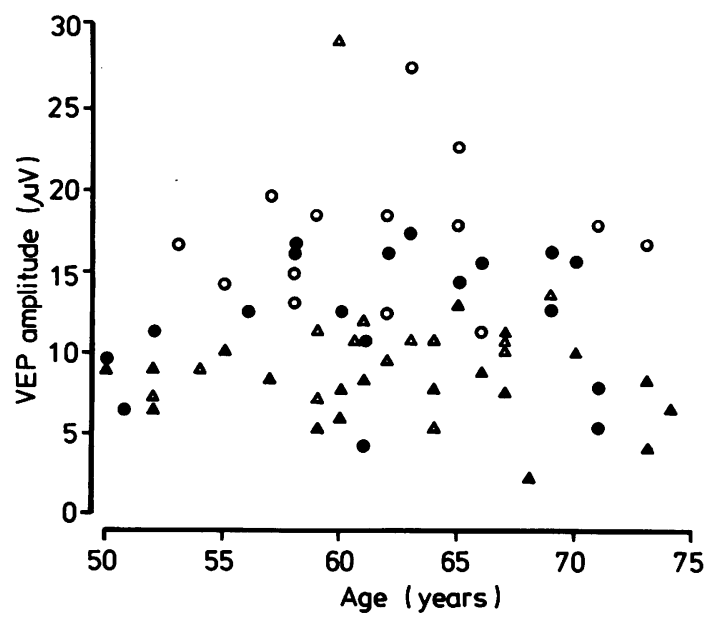

Fig 3 Scattergram of VEP amplitude with age. Stimulus checksize 50'. (Symbols as in fig 2.) 


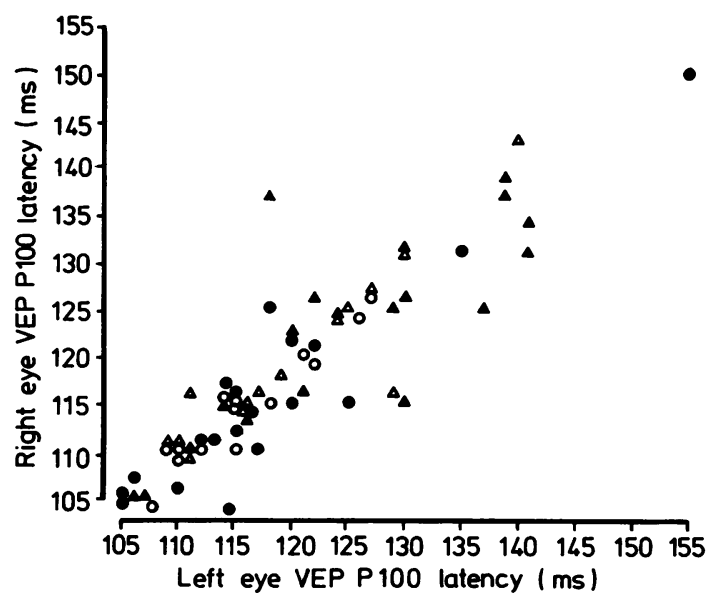

Fig 4 Scattergram of VEP P100 latency of the right eye with that of the left eye. Stimulus checksize 50'. (Symbols as in fig 2.)

were divided into those with and without chorea or into those on levodopa treatment and those not on levodopa treatment.

\section{Effects of age}

The association of age with the cortical and retinal potentials is shown in table 2. The VEP P100 and N150 latencies at both checksizes showed a positive correlation with age and these correlations (for example between VEP P100 latency to $50^{\prime}$ checksize and age) were strongest for Parkinson's disease patients $(\mathrm{r}=0.41 ; \mathrm{p}=0.006)$ and males $(\mathrm{r}=0.49 ; \mathrm{p}=0.002)$, but were also significant for control subjects $(\mathrm{r}=0.38 ; \mathrm{p}=0.023)$ and females $(\mathrm{r}=0.35$; $p=0.026$ ). Similarly partial correlation controlling for the effect of sex or the presence of Parkinson's disease did not alter these significant correlations between age and evoked potential. PERG implicit times increased with age but though these correlations were significant in the control group, the Parkinson's disease patients showed no change with age. However, the PERG amplitude of all subjects showed an inverse correlation with age at either checksizes, which was significant for Parkinson's disease patients at $50^{\prime}(\mathrm{r}=-0.51 ; \mathrm{p}=0.001)$ and at $25^{\prime}$ checksize $(r=-0.58 ; p<0.001)$. No significant effect of age was observed on the VEP amplitude at either checksize for any group, but there were consistent differences between the sexes at all ages (fig 3 ). Temporal dispersion (calculated as the difference in latency of the P100 and the N150) was not significantly correlated with age.

Interocular differences

There were more cases with a large interocular difference in the VEP P100 latency to 50' checks among the Parkinson's disease patients than among the control subjects (fig 4). There were eight Parkinson's disease patients whose P100 interocular difference was greater than $5 \mathrm{~ms}$ and in six of these the PERG PP1 implicit time interocular difference was also greater than $5 \mathrm{~ms}$. In five the cortical and retinal interocular differences were in the same direction and in one it was opposite.

\section{Discussion}

In this study we have demonstrated a significant difference between Parkinson's disease patients and well matched control subjects in the amplitude of VEP, PERG and flash ERG. A previous study in Parkinson's disease patients has also demonstrated reduced VEP amplitudes and increased interocular difference in VEP latency, ${ }^{4}$ but we have not shown the increased VEP latency in Parkinson's disease patients that has been reported elsewhere ${ }^{3-6}$ nor an increase in temporal dispersion. ${ }^{1}$ It has been suggested ${ }^{6}$ that the discrepancies between different reports on VEP in Parkinson's disease may be due to the greater sensitivity of grating patterns compared to checkerboard patterns and, if so, this might in part account for our normal Parkinson's disease VEP latencies. However, this study ${ }^{6}$ is somewhat unsatisfactory in that the grating subtense used is about one third of that for the checkerboard, and the retinal field stimulated is predominantly foveal for the grating whereas for the checkerboard it extends beyond the perimacula. This could explain the observed differences rather than the pattern form, per se. A recent report, ${ }^{35}$ which has failed to detect any VEP abnormalities in Parkinson's disease patients, is difficult to compare with previous studies because of the use of a large checksize and the fact that the control group consisted of "agecorrected" young normal subjects.

Discrepancies in visual acuity are unlikely to account for these VEP and PERG amplitude differences since Snellen chart testing did not reveal any significant differences in the visual acuity between Parkinson's disease patients and control subjects or between males and females. Moreover in those subjects with abnormal VEP or PERG to $50^{\prime}$ check stimulation, a similar though less marked difference was noted at $25^{\prime}$ checksize. If reduced acuity was responsible, the differences would have been more marked at the smaller checksize. The abnormality of the cortical and retinal potentials in the Parkinson's disease patients is likely to be due to the disease itself rather than drug therapy, since the changes were also noted in those on no medication. It is reported ${ }^{2}$ that the abnormality of the VEP is partly corrected by treatment and one might expect a difference in the 
VEP, or the PERG, between those with and without medication. We did not find any difference between these groups, though this may in part be due to the heterogeneity of the patients. Some did not receive treatment because they had only recently presented or were only mildly affected: in others with more severe or prolonged disease, major side-effects precluded the use of levodopa.

The significance of the reduced VEP amplitude in Parkinson's disease patients is highly dependent on the sex of the subject, since we observed a marked difference in amplitude between male and female subjects. Although the VEP amplitudes of Parkinson's disease males were low, they differed little from those of the control males. It is only in women that the VEP amplitude differed significantly between Parkinson's disease patients and control subjects and this accounts for the significant difference between Parkinson's disease patients and control subjects when both sexes are considered together. The difference between the VEP amplitude of males or females in our control subjects accords well with those previously reported. ${ }^{36}$ Although we observed a greater mean VEP latency in men than women, the difference was not statistically significant partly because of the wide scatter of results. We have confirmed the progressive delay in VEP latency that occurs with age and the absence of change in VEP amplitude with age, though there is a major difference in VEP amplitude between the sexes. ${ }^{35}$ In contrast to the VEP amplitude, the reduction in PERG amplitude in Parkinson's disease patients compared to control subjects was more marked in male than female subjects and for the flash ERGs there were no significant differences in amplitude between males and females. The PERG measurements show no significant differences between the sexes though like the VEP latency there is a change with age. The PERG implicit time increases progressively with age and the PERG amplitude falls.

The VEP, PERG and flash ERG originate from different parts of the retina and central nervous system and reflect different physiological processes. The changes in these potentials in Parkinson's disease may reflect the widespread nature of the biochemical disorder affecting both retina and central nervous system. Indeed Parkinson's disease patients have also been shown to have abnormal auditory evoked potentials. ${ }^{4}$ Alternatively, the reduction in the VEP may be secondary to the retinal abnormality itself and this hypothesis is supported by the association between the increased VEP latency and reduced PERG amplitude in Parkinson's disease patients, especially in male Parkinson's disease patients who show the most marked abnormality of cortical and retinal electrophysiology. Moreover, where there was a marked interocular difference in the VEP P100 latency, the direction of the difference was more commonly reflected in the interocular difference of the PP1 wave implicit time. Both VEP P100 latency and the PERG potentials showed similar changes with age; neither VEP latency nor PERG potentials showed significant differences between the sexes. The strength of these relationships between cortex and retina might be improved if it were possible to eliminate the influence of other sources of variation, for example skull thickness which may alter the VEP between males and females-whereas no such difference would be expected in the PERG between the sexes. Moreover, dopaminergic sites in the visual system proximal to the retina may also weaken the relationship of the VEP and PERG in Parkinson's disease patients. However, our findings do suggest that the PERG may be generated by similar retinal elements that are responsible at least in part for the VEP, that is to say the more proximal retinal layers including the ganglion cells.

That retinal disorders may affect the characteristics of the VEP invites caution in the use of VEPs to study central visual pathways. It is therefore important to exclude primary retinal pathology. The measurement of the PERG is simple and can be performed simultaneously with the VEP using the same stimuli. The PERG may have an important role in excluding retinal abnormalities as the cause of the VEP changes. Since dopamine is only one of a number of neurotransmitters found in the retina, it is possible that abnormal VEPs in other central nervous system disorders may in part be due to retinal abnormalities.

We are grateful to Professor DA Shaw, and Drs NEF Cartlidge and D Bates for permission to study the Parkinson's disease patients under their care and to the physicians and surgeons at the Royal Victoria Infirmary who permitted us to use their patients as control subjects. Karen Seed, Medical Physics Technician, performed many of the evoked potential recordings and we gratefully acknowledge her assistance.

\section{References}

${ }^{1}$ Bodis-Wollner I, Onofji M. System diseases and visual evoked potential diagnosis in neurology: changes due to synaptic malfunction. Ann NY Acad Sci 1982;388: 327-48.

${ }^{2}$ Bodis-Wollner I, Yahr MD. Measurement of visual evoked potentials in Parkinson's disease. Brain 1978;101:661-71.

${ }^{3}$ Bodis-Wollner I, Yahr MD, Thornton J. Visual evoked potentials and the severity of Parkinson's disease. In: Clifford Rose F, Capildeo R, eds. Progress in Parkinson's disease. New York: Pitman Medical, 1981:126-37. 
${ }^{4}$ Gawal MJ, Das P, Vincent S, Clifford Rose F. Visual and auditory evoked responses in patients with Parkinson's disease. J Neurol Neurosurg Psychiatry 1981;44:227-32.

${ }^{5}$ Kupersmith MJ, Shakin E, Siegel IM, Lieberman A. Visual system abnormalities in patients with Parkinson's disease. Arch Neurol 1982;39:284-6.

${ }^{6}$ Tartaglione A, Pizio N, Bino G, Spadavecchia L, Favale E. VEP changes in Parkinson's disease are stimulus dependent. J Neurol Neurosurg Psychiatry 1984;47: 305-7.

${ }^{7}$ Hakim AM, Mathieson G. Dementia in Parkinson's disease: a neuropathological study. Neurology 1979;29: 1209-14.

${ }^{8}$ Dyer RS, Howell WE, Macphail RC. Dopamine depletion slows retinal transmission. Exp Neurol 1981;71:326-40.

${ }^{9}$ Onofrj M, Bodis-Wollner I. Dopamine deficiency causes delayed visual evoked potentials in rats. Ann Neurol 1982;11:484-90.

${ }^{10}$ Shagass C. Pharmacology of evoked potentials in man. In: Psychopharmacology: a review of progress 1957-1967. Public Health Service Publication No 1836. Washington: US Government Printing Office, 1968:483-92.

${ }^{11}$ Ehinger B. Functional role of Dopamine in the retina. In: Osborne N, Chader G, eds. Progress in Retinal Research, Vol. 2. Oxford: Pergamon Press, 1983: 213-32.

${ }^{12}$ Iuvone PM, Galli CL, Garrison-Gund CK. Light activated tyrosine hydroxylase and increased dopamine synthesis in retinal amacrine neurones. Science 1978;202:901-2.

${ }^{13}$ Kramer SG. Dopamine: a retinal neurotransmitter: 1. Retinal uptake, storage and light-stimulated release of H3-dopamine in vivo. Invest Ophthalmol Vis Sci 1971;10:438-52.

${ }^{14}$ Kramer SG, Potts AM, Manganall Y. Dopamine: a retinal neurotransmitter: 2. Autoradiographic localization of H3-dopamine in the retina. Invest Ophthalmol Vis Sci. 1971;10:617-24.

${ }^{15}$ Economou SG, Stefanis CN. Changes of electrooculogram in Parkinson's disease. Acta Neurol Scand 1978;58:44-52.

${ }^{16}$ Cavallacci G, Perossini M. Interet clinique du test electroretinographique dans la maladie de Parkinson. J Franc Ophtalmol 1979;2:705-9.

${ }^{17}$ Dowling JE, Ehringer B, Heddon WL. The interplexiform cell; a new type of retinal neuron. Invest Ophthalmol Vis Sci 1976;15:916-26.

${ }^{18}$ Yonemura D, Kawasaki K. Electrophysiological study on activities of neuronal and non-neuronal retinal elements in man with reference to its clinical application. Jap J Ophthalmol 1978;22:195-213.

${ }^{19}$ Lawill T. The bar pattern electroretinogram for clinical evaluation of the central retina. Am J Ophthalmol 1974;78:121-6.

${ }^{20}$ Arden GB, Cater RM, Hogg CR, Powell DJ, Vaegan. Reduced Pattern Electroretinograms suggest a preganglionic basis for non-treatable human amblyopia.
J Physiol (Lond) 1980;308:82P-83P.

${ }^{21}$ Arden GB, Vaegan, Hogg CR. Clinical and experimental evidence that the pattern electroretinogram (PERG) is generated in more proximal retinal layers than the focal electroretinogram (FERG). Ann NY Acad Sci 1982;388: 580-601.

${ }^{22}$ Maffei L, Fiorentini A. Electroretinographic response to alternating gratings before and after section of the optic nerve. Science 1981;211:953-5.

${ }^{23}$ Arden GB. Vaegan, Hogg CR, Powell D, Carter RM. Pattern ERGs are abnormal in most amblyopes. Trans Ophthalmol Soc UK 1980;100:453-60.

${ }^{24}$ Fiorentini A, Maffei L, Pirchio M, Spinelli D, Porciatti V. The ERG in response to alternating gratings in patients with diseases of the peripheral visual pathway. Invest Ophthalmol Vis Sci 1981;21:490-3.

${ }^{25}$ Fiorentini A, Maffei L, Pirchio M, Poriatti V. Spinelli D. Pattern ERG in patients with unilateral alterations of the retinal ganglion cells. Doc Ophthalmol 1982;31: 131-3.

${ }^{26}$ Person HE, Wanger P. Pattern-reversal electroretinograms in squint amblyopia, artificial anisometropia and simulated eccentric fixation. Acta Ophthalmol 1982; 60:123-32.

${ }^{27}$ Sherman J. Simultaneous pattern-reversal electroretinograms and visual evoked potentials in disease of the macula and optic nerve. Ann NY Acad Sci 1982;388:214-25.

${ }^{28}$ Sokol S, Nadler D. Simultaneous electroretinograms and visual evoked potentials from adult amblyopes in response to a patterned stimulus. Invest Ophthalmol Vis Sci 1979;18:848-55.

${ }^{29}$ Vaegan, Arden GB, Hogg CR. Properties of normal electroretinograms evoked by patterned stimuli in man. Doc Ophthalmol 1982;31:111-29.

${ }^{30}$ Armington JC, Corwin RT, Marsetta R. Simultaneously recorded retinal and cortical responses to patterned stimuli. J Opt Soc Am 1971;61:1514-15.

${ }^{31}$ Armington JC. Simultaneous electroretinograms and evoked potentials. Ann NY Acad Sci 1982;388:572-9.

${ }^{32}$ Sokol S, Jones K, Nadler D. Comparison of the spatial response properties of the human retina and cortex as measured by simultaneously recorded ERGs and VEPs. Vis Res 1983;23:723-7.

${ }^{33}$ Mitchell KW, Howe JM. Parallel pattern-generated electroretinogram and visual evoked cortical potential. Doc Ophthalmol 1984;40:69-77.

34 Jasper HH. Report to the committee on methods of examination in electroencephalography. Electroencephalogr Clin Neurophysiol 1958;10:370-5.

${ }^{35}$ Dinner DS, Luders H, Hanson M, Lessor RP, Klem G. Pattern evoked potentials (PEPs) in Parkinson's disease. Neurology 1985;35:610-3.

${ }^{36}$ Halliday AM, Barrett G, Carroll WM, Kriss A. Problems in defining the normal limits of the VEP. In: Courjon J, Mauguiere F, Revol M, eds. Clinical Applications of Evoked Potentials in Neurology. New York: Raven Press, 1982:1-9. 